Revista peruana de biología 26(4): 461 - 468 (2019) doi: http://dx.doi.org/10.15381/rpb.v26i4.16238 ISSN-L 1561-0837; eISSN: 1727-9933

Universidad Nacional Mayor de San Marcos

\section{Revisión taxonómica de la rana marsupial Gastrotheca peruana (Amphibia: Hemiphractidae) en base al gen mitocondrial $16 \mathrm{~S}$}

\section{TRABAJOS ORIGINALES}

$\begin{array}{ll}\text { Presentado: } & 31 / 05 / 2019 \\ \text { Aceptado: } & 18 / 11 / 2019 \\ \text { Publicado online: } & 16 / 12 / 2019\end{array}$

\section{Correspondencia:}

*Autor de correspondencia

Daniel Rodríguez: daal911@yahoo.com Guillermo D’Elía: guille.delia@gmail.com Hernán Ortega: tortegat@unmsm.edu.pe César Aguilar: caguilarp@unmsm.edu.pe

1 y 3 Av. Arenales 1256, Jesús María, Lima, Perú. 2 Campus Isla Teja s/n, Valdivia, Chile.

Otros datos de los autores / biografía: Daniel Rodríguez: 0000-0002-3324-6555 Guillermo D'Elía: 0000-0001-7173-2709 Hernán Ortega: 0000-0002-4396-2598 César Aguilar: 0000-0001-6372-7926

\section{Citación:}

Rodríguez D., G. D’Elía, H. Ortega, C. Aguilar. 2019. Revisión taxonómica de la rana marsupial Gastrotheca peruana (Amphibia: Hemiphractidae) en base a el gen mitocondrial 16S. Revista peruana de biología 26(4): 461 - 468 (Diciembre 2019). doi: http://dx.doi.org/10.15381/rpb.v26i4.16238

Palabras clave: taxonomía; Perú; filogeografía; Andes; rana marsupial; biodiversidad. Keywords: taxonomy; Peru; phylogeography; Andes; marsupial frog; biodiversity.

\section{Taxonomic evaluation of the marsupial frog Gastrotheca peruana (Amphibia: Hemiphractidae) based on mitochon- drial 16S rRNA gene}

\author{
Daniel Rodríguez*1 ${ }^{*}$ Guillermo D’Elía ${ }^{2}$, Hernán Ortega ${ }^{3}$, César \\ Aguilar $^{1}$
}

1 Universidad Nacional de Mayor de San Marcos, Museo de Historia Natural, Departamento de Herpetología, Perú.

2 Instituto de Ciencias Ambientales y Evolutivas, Facultad de Ciencias, Universidad Austral de Chile, Chile.

3 Universidad Nacional de Mayor de San Marcos, Museo de Historia Natural, Departamento de Ictiología, Perú.

\section{Resumen}

Se revisa el estado taxonómico de Gastrotheca peruana usando métodos filogenéticos en base a secuencias de $16 \mathrm{~S}$ rRNA. Los árboles de máxima verosimilitud y Bayesiano mostraron que las variantes génicas de $G$. peruana forman dos clados que no son hermanos. Uno de estos clados se distribuye en el norte de Perú, incluyendo un individuo procedente de la localidad típica de G. peruana dissimilis. El segundo clado está restringido al centro de Perú y contiene individuos de las localidades tipo de dos formas nominales, G. p. peruana y G. $p$. junensis, y es hermano de G. aratia. De esta forma, reconocemos dos especies dentro de lo que actualmente se conoce como G. peruana. Restringimos Gastrotheca peruana a las poblaciones del centro de Perú (departamentos de Ancash, Lima, Pasco y Junín) y asignamos Gastrotheca dissimilis a las poblaciones de los departamentos de La Libertad y Cajamarca.

\section{Abstract}

The taxonomic status of Gastrotheca peruana is evaluated using phylogenetic methods and $16 \mathrm{~S}$ gene sequences. The maximum likelihood and Bayesian trees showed that the genetic variants of $G$. peruana form two clades that are not sister to each other. One of these clades is present in northern Perú and includes a specimen collected at the type locality of G. peruana dissimilis. The other clade is restricted to central Peru and contains individuals from type localities of two nominal forms, G. p. peruana and G. p. junensis, and is sister to G. aratia. Our results suggest that $G$. peruana sensu lato currently encompass two species. We restrict Gastrotheca peruana to populations from central Perú (departments of Ancash, Lima, Pasco and Junín) and assign populations from departments of La Libertad and Cajamarca to Gastrotheca dissimilis.

\section{Introducción}

Las especies del género Gastrotheca son conocidas como ranas marsupiales porque las hembras presentan una bolsa de reproducción cerrada ubicada en el dorso donde alojan a los embriones (Duellman \& Fritts 1972, Duellman 2015). Son conocidas 70 especies de Gastrotheca, distribuidas en Costa Rica, Panamá, las regiones andinas de Ecuador, Colombia, Perú, Bolivia y Argentina, en la cuenca alta de la Amazonía, en el este de Venezuela, en el escudo de Guyana y en los bosques costeros del Atlántico en Brasil (Blackburn \& Duellman 2013, Duellman 2015, Duellman \& Venegas 2016, Frost 2019). El primer estudio filogenético del género Gastrotheca fue el de Wiens et al. (2007), donde se reconocen 46 especies utilizando tres marcadores mitocondriales y dos nucleares; los resultados presentaron al 
género Gastrotheca como monofilético. Posteriormente Blackburn y Duellman (2013) reportan 56 especies empleando dos marcadores mitocondriales y dos nucleares; sus resultados indicaron que el género es monofilético y contiene seis clados ampliamente distribuidos; dos de estos ubicados en los Andes del noreste y centrales y contienen la mayor diversidad de especies (46), de las cuales (14) se distribuyen en Perú.

En Perú se conocen 29 especies de Gastrotheca (Duellman \& Venegas 2016, Frost 2019). Estas especies han sido caracterizadas en base a la morfología, coloración corporal, modo de desarrollo larval, osteología craneal y caracteres moleculares (Boulenger 1900, Barbour \& Noble 1920, Vellard 1957, Duellman \& Fritts 1972, Trueb \& Duellman 1978, Duellman 1987, Duellman \& Hillis 1987, Duellman et al. 2001, Duellman et al. 2004, Duellman \& Venegas 2005, Duellman et al. 2006, Catenazzi \& Lehr 2009, Catenazzi \& von May 2011, Duellman et al. 2011, Lehr \& Catenazzi 2011, Duellman 2013, Duellman \& Venegas 2016).

Una de las especies de Gastrotheca que se distribuye en el Perú es Gastrotheca peruana (Boulenger 1900); su localidad tipo se ubica en el distrito de Caraz, provincia de Huaylas, departamento de Ancash y es parte del clado "Andes centrales" junto con otras 22 especies (Boulenger 1900, Blackburn \& Duellman 2013). Gastrotheca peruana sensu lato se distribuye hacia el sur de la localidad tipo hasta la región de Junín, en la Cordillera Central a elevaciones de 4000 - $4600 \mathrm{~m}$ en la cabecera de la cuenca del rio Mantaro y vertiente del Nudo de Pasco. Hacia el norte de la localidad tipo llega a Cajamarca, en la Cordillera Occidental a elevaciones de 2300 - $3350 \mathrm{~m}$ en la parte alta del valle del río Marañón y 2300 - 3520 m en la vertiente del Pacífico (Boulenger 1900, Vellard 1957, Duellman \& Fritts 1972, Duellman \& Venegas 2005, Aguilar et al. 2007, Duellman et al. 2014, Duellman 2015).

Investigaciones previas sugieren que $G$. peruana tal cual se delimita actualmente puede ser un complejo de subespecies y especies (Vellard 1957, Duellman 2015). Vellard (1957) planteó que G. peruana está dividida en tres subespecies, la forma $G$. p. peruana que se distribuye desde la localidad tipo hacia el norte hasta la región de Cajamarca, G. p. dissimilis que se distribuye al norte de la localidad tipo en la región de La Libertad y $G$. p. junensis al sur de la localidad tipo en la región de Pasco. El lago de Punrún, localidad tipo de G. p. junensis, se localiza en la región de Pasco (Cosme \& Silva 2016). El reconocimiento de estas formas se basó en diferencias de índices cefálicos, longitud de las patas y patrón de pustulación en la piel dorsal. Duellman (2015) estudiando la variación geográfica de 36 especímenes de $G$. peruana procedentes de Cajamarca, Ancash, Huánuco y Junín, encontró que la longitud hocico cloaca (LHC), ancho de la cabeza (AC), ancho del párpado (AP), longitud del pulgar (LPU) y diámetro del ojo (DO), eran mayores en especímenes de poblaciones de Cajamarca y Ancash que en los individuos de Huánuco y Junín, sugeriendo que un análisis con tecnicas moleculares podria revelar especies crípticas. En el presente estudio mediante analisis de variación del gen $16 \mathrm{~S}$ rRNA se evaluó el grado de diferenciación entre individuos de dis- tintas localidades, incluyendo de la localidad típica, asignados a Gastrotheca peruana.

\section{Materiales y métodos}

Material biológico.- Se examinaron 11 especímenes depositados en la colección científica del Departamento de Herpetología del Museo de Historia Natural de la Universidad Nacional Mayor de San Marcos (MUSM), seis de ellos colectados recientemente fueron asignados a Gastrotheca peruana, procedentes de la localidad tipo en Caraz (MUSM 34886), región de Pasco (MUSM 34909), Cajamarca (MUSM 32773), La Libertad (MUSM 32123), Ancash (MUSM 32789) y Lima (MUSM 34880). Los cinco especímenes restantes pertenecen a Gastrotheca phelloderma (MUSM 33350), Gastrotheca marsupiata (MUSM 33444), Gastrotheca sp. 1 (MUSM 33319), Gastrotheca sp. 2 (MUSM 32794) y Pleurodema marmoratum (MUSM 33449); los dos ejemplares asignados como sp. provienen de localidades poco muestreadas y presentan caracteres morfológicos que las diferencian de otras especies conocidas de tales localidades. Pleurodema marmoratum fue usada como grupo externo (ver abajo). Los ejemplares depositados en la colección fueron preservados en formaldehido al 10\% y almacenados al 70\% en alcohol etílico (McDiarmid 1994), mientras que sus tejidos fueron fijados en alcohol al 96\% y depositados en la colección del Departamento de Herpetología del Museo de Historia Natural de la Universidad Nacional Mayor de San Marcos (MUSM).

\section{Análisis Filogenético}

Generación de secuencias de ADN.- Se extrajo el ADN genómico de muestras de hígado. Porciones de los tejidos extraídos fueron procesados empleando el equipo de extracción Wizard ${ }^{\circledR}$ SV Genomic DNA Purification System (Promega (C), siguiendo el protocolo indicado por el fabricante. Se amplificó un fragmento del gen 16S vía PCR usando los iniciadores 16sar-F (CGCCTGTTTATCAAAAACAT) y 16sbr-R (CCGGTCTGAACTCAGATCACGT); el segundo difiere en una base (A por S) del reportado por (Wiens et al. 2007) y siguiendo el protocolo de amplificación: desnaturalización inicial a $94{ }^{\circ} \mathrm{C}$ por 2 minutos, 35 ciclos de desnaturalización a $94{ }^{\circ} \mathrm{C}$ por 30 segundos, alineamiento a $57^{\circ} \mathrm{C}$ por 30 segundos, extensión a $72{ }^{\circ} \mathrm{C}$ por 2 minutos y una extensión final a $72{ }^{\circ} \mathrm{C}$ por 10 minutos. La limpieza y secuenciación de los amplicones (producto del PCR) se hizo en un servicio externo (Macrogen, Inc., Seoul, South Korea). Las 11 secuencias obtenidas fueron editadas usando CodonCodeAligner v.6.0.2 (Genecodes Corporation $($ ) .

Inferencia filogenética.- Las 11 secuencias generadas se integraron en una matriz con otras 12 secuencias obtenidas de Genbank. De estas últimas, dos pertenecen a Gastrotheca peruana, especímenes procedentes de Cajamarca (KU 181740) y Junín (KU 207815); el resto de las secuencias corresponde a otras especies de Gastrotheca que se distribuyen por los Andes del noreste y centrales de Perú y sur de Ecuador (Blackburn \& Duellman 2013). Las 23 secuencias fueron alineadas usando el software MUSCLE (Edgar 2004); el alineamiento fue inspeccionado visualmente con MEGA 7 versión 7.0.14 (Kumar et al. 1994). El 
modelo evolutivo de sustitución nucleotídica que se ajustó a los datos, según el criterio de información bayesiana (BIC por sus siglas en inglés) implementado en jModelTest v.2.1.7 (Darriba et al. 2012), fue TIM2+G (estimación de parámetros: $-\operatorname{lnL}=2253.6383, \mathrm{p}=51$ ). Se realizó un análisis de Máxima Verosimilitud (ML) en CIPRES Science Gateway (Miller et al. 2010) con RAxML 8.2.10 (Stamatakis 2014), usando el modelo general de tiempo reversible con sitios invariables y distribución gamma $(\mathrm{GTR}+\mathrm{I}+\Gamma$; $-\operatorname{lnL}=$ 2251.0102, p= 54), dado que el modelo TIM2+G no está implementado en RAxML. El soporte relativo de los clados se evaluó con 1000 réplicas de Bootstrap.

La inferencia bayesiana (B) fue realizada en MrBayes 3.2.6 (Huelsenbeck \& Ronquist 2001), y el modelo TIM2+G fue reemplazado por el modelo GTR $+\mathrm{I}+\Gamma$ dado que el primero no se puede implementar en dicho programa (ver un escenario similar en Lecocq et al. 2013). El análisis incluyó cuatro cadenas de Markov Monte Carlo ( $\mathrm{mcmc}$ ), que se llevaron adelante por 20 millones de generaciones, las que fueron muestreadas cada 1000 generaciones. El primer $25 \%$ de los datos muestreados fue descartado y se retuvieron aquellos árboles y valores de parámetros de la zona de convergencia. Para la interpretación de inferencias filogenéticas, consideramos como evidencia de un buen apoyo soportes de Bootstrap (BS) mayores a 70\% y probabilidades posteriores (PP) mayores al 95\% (e.g., Blackburn \& Duellman 2013, Quyen et al. 2015, Wang et al. 2015, Luza et al. 2015, Huang et al. 2016, Song et al. 2016).

\section{Resultados}

Inferencia filogenética.- En las 23 secuencias de 502 pares de bases se identificaron 23 haplotipos, uno de ellos proveniente de la localidad tipo de Gastrotheca peruana (Tabla 1). Los resultados filogenéticos muestran que las secuencias de Gastrotheca peruana forman dos clados principales que cuentan con buen apoyo. El primer clado $(B S=76$; $\mathrm{PP}=0.99)$ está formado por haplotipos de especímenes provenientes de las regiones de Cajamarca (provincias de Celendin y Hualgayoc) y la Libertad (provincia Sánchez Carrión). El segundo clado (BS= 94; $P P=1)$ está formado por haplotipos de especímenes provenientes de Ancash (provincias de Huaylas y Bolognesi), Lima (provincia Oyón), Pasco (provincia Daniel Alcides Carrión) y Junín (provincia de Junín) (Figs. 1 y 2).

El clado 1 (Cajamarca y La Libertad) se distribuye por la Cordillera Occidental a elevaciones altas (3294-4000 m) en el valle del río Marañón y Nudo de Mollepata; el clado 2 (Ancash, Lima, Pasco y Junín) por la Cordillera Occidental a elevaciones altas (3726 - $4388 \mathrm{~m})$ en el valle del río Santa y la Cordillera de Huayhuash en la vertiente del Pacífico y por la Cordillera Central (4000 - $4115 \mathrm{~m}$ ) en la cabecera de cuenca del río Mantaro y Nudo de Pasco. Ambos clados no son hermanos. El clado 2 es hermano de G. aratia, una especie que morfológicamente se diferencia de $G$. peruana y se distribuye a elevaciones bajas ( 2560 - $2875 \mathrm{~m}$ ) en el valle del río Chatano, un tributario del río Chamaya que llega al río Marañón. La localidad tipo de G. aratia se encuentra en Cajamarca

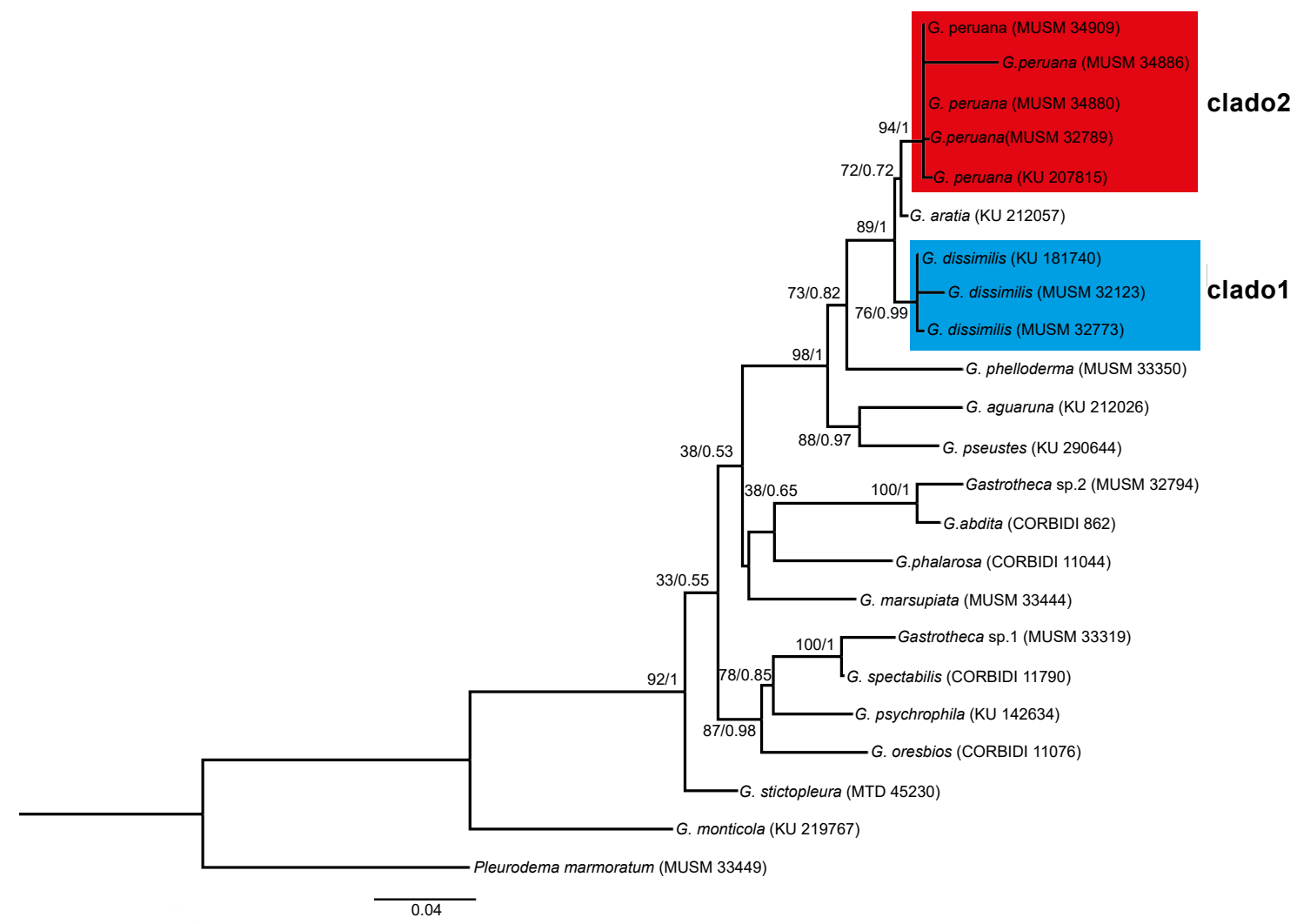

Figura 1. Topología del árbol filogenético derivado de haplotipos del gen 16S de Gastrotheca. Se presenta los valores de soporte Bootstrap y probabilidad posteriores (BS/PP) 


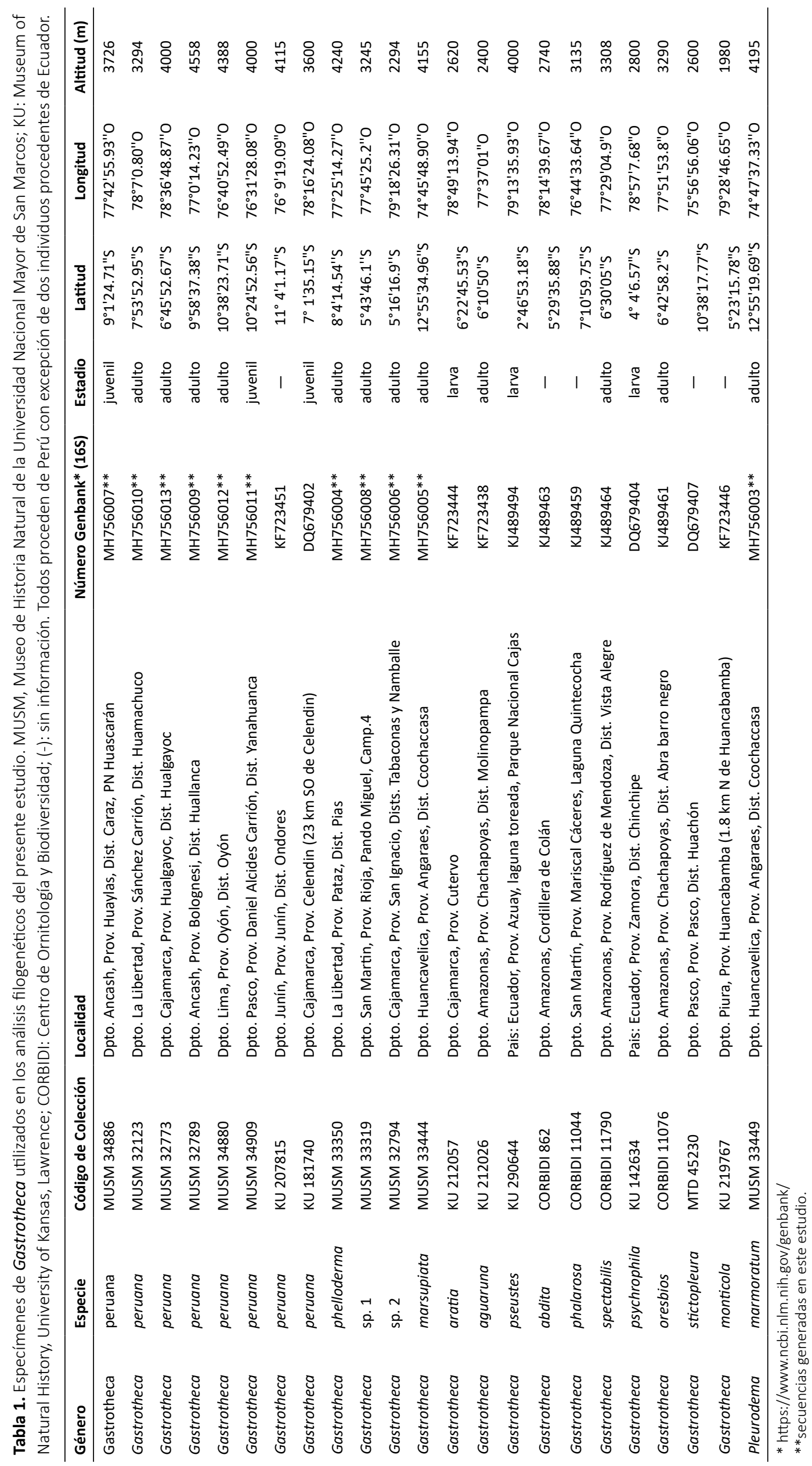




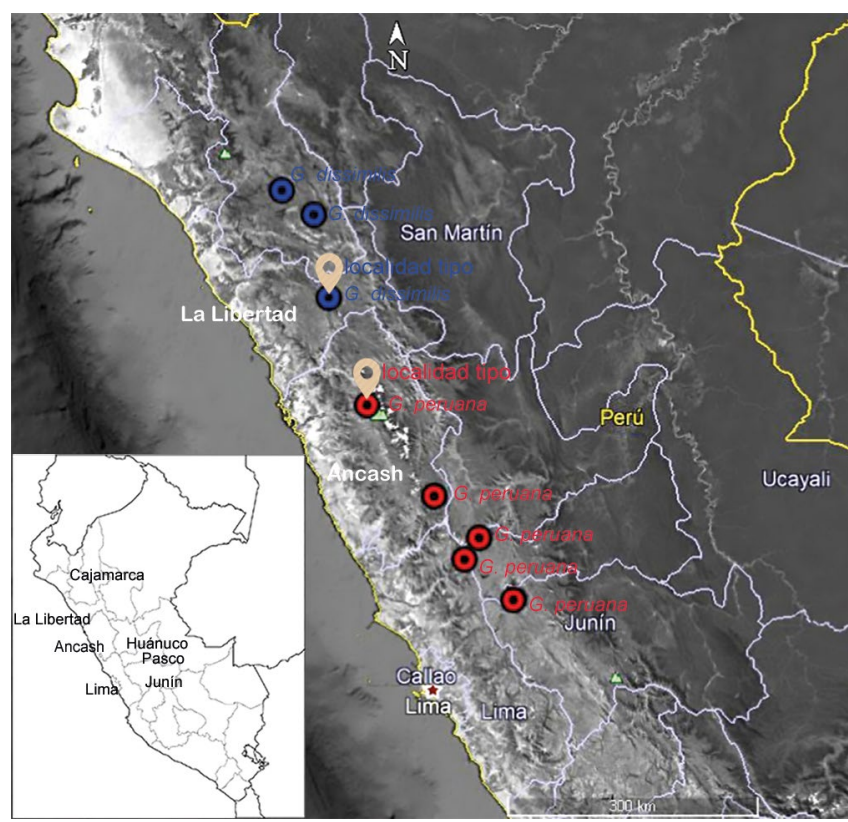

Figura 2. Distribución geográfica de los clados de Gastrotheca peruana sensu lato; azul: clado 1 o G. dissimilis (Cajamarca y La Libertad); rojo: clado 2 o G. peruana sensu stricto (Ancash, Lima, Pasco y Junín).

(provincia Cutervo) que está ubicada más al norte de las localidades registradas para los clados 1 y 2 (Duellman et al. 2014). El grupo monofilético formado por el clado 2 de $G$. peruana y $G$. aratia tiene apoyo moderado $(B S=72$; $\mathrm{PP}=0.72$ ). El clado 1 de $G$. peruana es hermano de este grupo formando un clado más inclusivo con valores alto de soporte $(\mathrm{BS}=89 ; \mathrm{PP}=1)$.

Gastrotheca sp. 1 (MUSM 33319) de San Martin (provincia Rioja) aparece como hermana a $G$. spectabilis en una relación que tiene apoyo alto $(\mathrm{BS}=100 ; \mathrm{PP}=1)$; mientras que $G$. sp. 2 (MUSM 32794) de Cajamarca (provincia San Ignacio) es hermana de $G$. abdita en una relación fuertemente apoyada ( $\mathrm{BS}=100 ; \mathrm{PP}=1)$. La formalización de estas dos especies candidatas como nuevas especies queda pendiente a la corroboración de su distinción mediante análisis más detallados que deben incluir una serie mayor de especímenes y evidencia morfológica.

\section{Discusión}

Delimitación de especies.- Las especies son utilizadas como elementos de análisis en diversas disciplinas tales como ecología, biogeografía y la biología de la conservación (Navas et al. 2013, Catenazzi et al. 2013, Capinha et al. 2017). Debido a que los límites de las especies deben tratarse como hipótesis pasibles de ser puestas a prueba (Fitzhugh 2005, Yeates et al. 2011, Aguilar et al. 2013), cada método empleado, con su respectivo conjunto de datos, corrobora o falsifica determinado esquema de delimitación.

Nuestros resultados, basados en un marcador mitocondrial, sugieren que Gastrotheca peruana representa dos linajes de nivel específico. El primer linaje (clado 1 en Figs. 1 y 2) se distribuye por las regiones de Cajamarca y La Libertad, para esta última región se incluye la provincia de Huamachuco de donde proviene el ejemplar analizado (MUSM 32123) y que es el lugar de registro del lectotipo de Gastrotheca peruana dissimilis (Vellard 1957, Morales et al. 1990). De esta forma, los especímenes de Gastrotheca peruana que se distribuyen por las regiones de este clado se asignan a $G$. dissimilis, la que es aquí reconocida a nivel de especie. El segundo linaje (clado 2 en Figs. 1 y 2) tiene como distribución las regiones de Ancash, Lima, Junín y Pasco. Entre los especímenes analizados en el clado 2 se incluye un ejemplar (MUSM 36589) topotípico de G. peruana (i.e., colectado en la localidad tipo: distrito de Caraz, región Ancash) y el ejemplar (MUSM 34909) topotípico de Gastrotheca peruana junensis. Por lo tanto, las poblaciones de Gastrotheca peruana que se distribuyen por las regiones de este segundo grupo monofilético son asignados a Gastrotheca peruana sensu stricto.

Vellard (1957) en base a diferencias en índices cefálicos, longitud de las patas y patrón de pustulación en la piel dorsal, planteó que $G$. peruana está dividida en tres subespecies (Fig. 3). En el presente estudio, los resultados basados en secuencias de ADN, no corroboran la hipótesis de Vellard (1957). Los especímenes examinados de ambos clados pueden ser diferenciados morfológicamente en base a ocho caracteres (Fig. 4): 1) textura de la piel dorsal, 2) diseño del tímpano, 3) tubérculos ventrolaterales a la cloaca, 4) longitud de separación entre el tímpano-ojo, 5) fórmula de la membrana en los dedos del pie, 6) porcentaje de la longitud de la tibia en relación a la longitud hocico cloaca, 7) porcentaje de la longitud del pie en relación a la longitud hocico cloaca y 8) patrón de color del iris (en vida). Duellman (2015) encontró cinco variables morfométricas que diferencian dos morfototipos de G. peruana s.l.; un morfotipo corresponde a las poblaciones Cajamar-

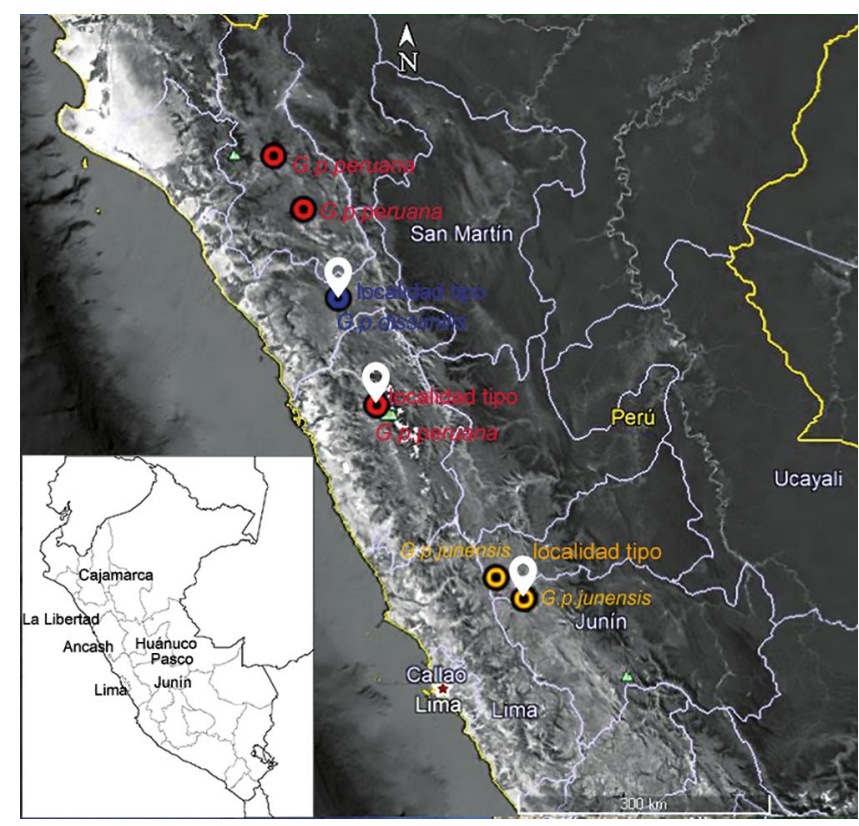

Figura 3. Distribución geográfica de las tres subespecies de Gastrotheca peruana propuestas por Vellard (1957). Rojo: G. peruana peruana (Ancash y Cajamarca). Azul: G. peruana dissimilis (La Libertad). Naranja: G. peruana junensis (Pasco y Junín). 

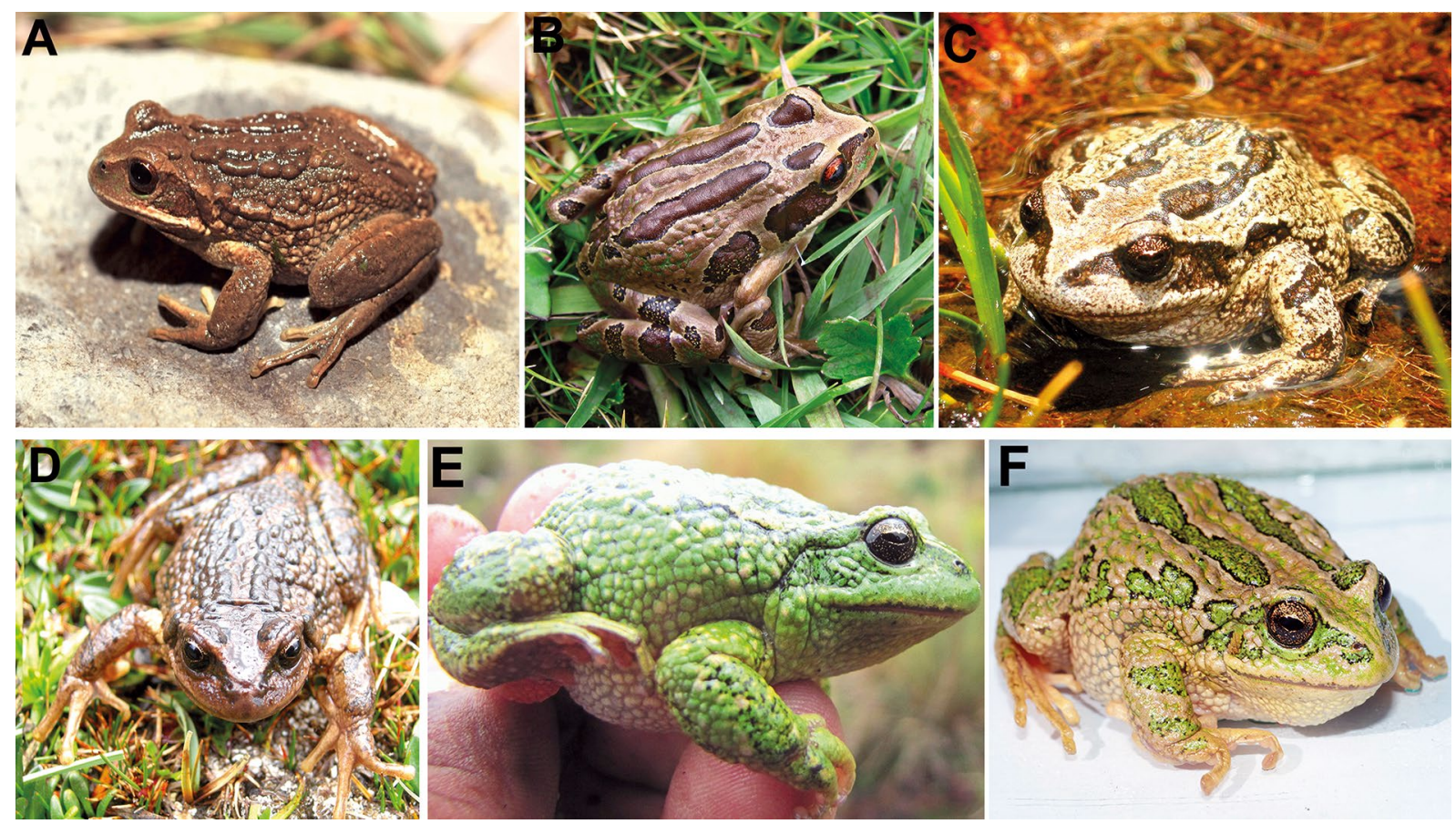

Figura 4. Especímenes de Gastrotheca peruana sensu lato: A-B. G. dissimilis: (A) MUSM 36678, macho, 47.83 mm longitud hocico cloaca (LHC), Hualgayoc, Hualgayoc, Cajamarca, foto cortesía de J. Cusi; (B) MUSM 32123, hembra, 43.59 mm longitud hocico cloaca (LHC), Huamachuco, Sánchez Carrión, La Libertad, foto cortesía de A. Ticona. C-F. G. peruana sensu stricto: (C) MUSM 36589, hembra, 45.37 mm longitud hocico cloaca (LHC), Caraz, Huaylas, Ancash (localidad tipo); (D) MUSM 34880, macho, 41.87 mm longitud hocico cloaca (LHC), Oyón, Oyón, Lima; (E) MUSM 31244, hembra, 47.65 mm longitud hocico cloaca (LHC), Paucartambo, Pasco, Pasco, foto cortesía de A. Mendoza; (F) no colectado, hembra, $45.97 \mathrm{~mm}$ longitud hocico cloaca (LHC), zona de amortiguamiento de la Reserva Nacional de Junín, Carhuamayo, Junín, Junín, foto cortesía de L. Castillo.

ca-Ancash y el otro a aquellas de Huánuco-Junín; esta evidencia le permitió sugerir que análisis moleculares podría revelar la existencia de especies crípticas dentro de $G$. peruana s.l. Nuestros estudios, basados en secuencias de ADN, corroboran la hipótesis de Duellman (2015) y nos permiten decir que $G$. dissimilis es una especie distinta de G. peruana s.s.. Estudios adicionales en progreso por el primer autor pondrán a prueba esta hipótesis.

Distribución, historia natural y estado de conservación de Gastrotheca peruana.- Actualmente G. peruana s.l. se encuentra como categoría de "preocupación menor" en la lista de especies amenazadas de la Unión Internacional para la Conservación de la Naturaleza, debido a su distribución en un amplio rango de hábitats y presuntos tamaños poblacionales grandes y estables (IUCN SSC ASG 2018). A raíz de los resultados del presente estudio, en que se reconocen dos especies, el grado de amenaza de $G$. peruana y $G$. dissimilis debe ser evaluado a la luz de las distribuciones conocidas de ambas especies, que incluyen áreas bajo concesión minera en las regiones de Cajamarca, La Libertad y Ancash (Aguilar et al. 2012). El nuevo espécimen de Gastrotheca peruana registrado y examinado del distrito de Caraz, fue encontrado fuera del Parque Nacional Huascarán; otros especímenes fueron observados en la zona de amortiguamiento y al interior del área protegida; el espécimen examinado fue encontrado debajo de piedras, rocas y a orillas del riachuelo y se encuentran en sintopía con la lagartija Liolae- mus chavin (Liolaemidae) (Aguilar et al. 2013).

La diversidad de especies del género Gastrotheca en el Perú está siendo subestimada y las amenazas antrópicas son evidentes fuera de áreas naturales protegidas (Aguilar et al. 2010), es necesario por tanto promover su conservación evaluando la tendencia poblacional de las especies de Gastrotheca con énfasis en aquellas endémicas de Perú.

\section{Literatura citada}

Aguilar C., M. Lundberg, K. Siu Ting, et al. 2007. Nuevos registros para la herpetofauna del departamento de Lima, descripción del renacuajo de Telmatobius rimac Schmidt, 1954 (Anura: Ceratophrydae) y una clave de los anfibios. Revista Peruana de Biología, 14(2): 209-216. https://doi.org/10.15381/rpb.v14i2.1736

Aguilar, C., C. Ramírez, D. Rivera, et al. 2010. Anfibios andinos del Perú fuera de Áreas Naturales Protegidas: amenazas y estado de conservación. Revista Peruana de Biología,17(1): 5-28. https://doi.org/10.15381/rpb. v17i1.46

Aguilar C., R. Gamarra, C. Ramírez, et al. 2012. Anfibios andinos y estudios de impacto ambiental en concesiones mineras de Perú. Alytes, 29 (1-4): 88-102.

Aguilar C., P. L. Wood Jr, J.C. Cusi et al. 2013. Integrative taxonomy and preliminary assessment of species limits in the Liolaemus walkeri complex (Squamata, Liolaemidae) with descriptions of three new species from Peru. Zookeys, 364: 47-91. https://doi.org/10.3897/ zookeys.364.6109 
Barbour T., \& G. K. Noble. 1920. Some amphibians from northwestern Peru, with a revision of the genera Phyllobates and Telmatobius. Bulletin of the Museum of Comparative Zoology Cambridge, Massachusetts 63: 395-427.

Blackburn D. C. \& W. E. Duellman. 2013. Brazilian marsupial frogs are diphyletic (Anura: Hemiphractidae: Gastrotheca). Molecular Phylogenetics and Evolution, 68: 709-714. https://doi.org/10.1016/j.ympev.2013.04.021

Boulenger G. A. 1900. Descriptions of new batrachians and reptiles collected by Mr. P. O. Simons in Peru. The Annals and magazine of natural history; zoology, botany, and geology (7) 6: 181-186. https://doi. org/10.1080/00222930008678355

Capinha C., H. Seebens, P. Cassey, et al. 2017. Diversity, biogeography and the global flows of alien amphibians and reptiles. Diversity and Distributions 1-10. https://doi. org/10.1111/ddi.12617

Catenazzi A. \& E. Lehr. 2009. The generic allocation of "Hyla" antoniiochoai De la Riva \& Chaparro, 2005 (Anura), with description of its advertisement call and ecology. Zootaxa 2304: 61-68. https://doi.org/10.11646/zootaxa.2304.1.5

Catenazzi A. \& R. Von May. 2011. New species of marsupial frog (Hemiphractidae: Gastrotheca) from an isolated montane forest in Southern Peru. Journal of Herpetology 45(2): 161-166. https://doi.org/10.1670/10-070.1

Catenazzi A., E. Lehr \& V. T. Vredenburg. 2013. Thermal physiology, disease, and amphibian declines on the eastern slopes of the Andes. Conservation Biology, 28(2): 1-9. https://doi.org/10.1111/cobi.12194

Cosme W. \& J. Silva. 2016. Ecosistema de la laguna Punrun, estudio de biodiversidad del ecosistema y análisis de la calidad del agua. Asociación civil centro de cultura popular labor. Sonimágenes del Perú SCRL, Pasco, Perú. $123 \mathrm{pp}$.

Darriba D., G. L. Taboada, R. Doallo, et al. 2012. "jModelTest 2: more models, new heuristics and parallel computing". Nature Methods 9(8): 1-2. https://doi.org/10.1038/ nmeth.2109

Duellman W. E.1987. Two new species of marsupial frogs (Anura: Hylidae) from Peru. Copeia. 4: 903-909. https:// doi.org/10.2307/1445553

Duellman W. E. 2013. An elusive new species of marsupial frog (Anura: Hemiphractidae: Gastrotheca) from the Andes of northern Peru. Phyllomedusa 12(1): 3-11. https:// doi.org/10.11606/issn.2316-9079.v12i1p3-11

Duellman W. E. 2015. Marsupial frogs: Gastrotheca \& allied genera Marsupial Frogs: Gastrotheca and Allied Genera. John Hopkins University Press, ISBN 978-1-42141675-5. 408pp.

Duellman W. E. \& T. Fritts. 1972. A taxonomic review of the southern Andean marsupial frogs (Hylidae: Gastrotheca). Occasional Papers of the Museum of Natural History Museum the University of Kansas, Lawrence, Kansas 9: 1-37. https://doi.org/10.5962/bhl. part.15140

Duellman W. E. \& D. M. Hillis. 1987. Marsupial frogs (Anura: Hylidae: Gastrotheca) of the Ecuadorian Andes: Resolution of taxonomic problems and phylogenetic relationships. Herpetologica, 43 (2): 141-173.
Duellman W. E. \& P. Venegas. 2005. Marsupial frogs (Anura: Hylidae: Gastrotheca) from the Andes of northern Peru with descriptions of two New Species. Herpetologica, 3: 295-307. https://doi.org/10.1655/04-105.1

Duellman W. E. \& P. Venegas. 2016. Diversity of marsupial frogs (Anura: Hemiphractidae: Gastrotheca) in the northern cordillera central, Peru, with the descriptions of two new species. Phyllomedusa 15(2): 103-117. https:// doi.org/10.11606/issn.2316-9079.v15i2p103-117

Duellman W. E., A. J. Barley \& P. Venegas. 2014. Cryptic species diversity in marsupia frogs (Anura: Hemiphractidae: Gastrotheca) in the Andes of northern Peru. Zootaxa 3768 (2): 159-177. https://doi.org/10.11646/zootaха.3768.2.4

Duellman W. E., A. Catenazzi \& D. C. Blackburn. 2011. A new species of marsupial frog (Anura: Hemiphractidae: Gastrotheca) from the Andes of southern Peru. Zootaxa 3095: 1-14. https//doi.10.5281/zenodo.207642

Duellman W. E., E. Lehr \& C. Aguilar. 2001. A new species of marsupial frog (Anura: Hylidae: Gastrotheca) from the Cordillera Azul in Peru. Occasional Papers of the Museum of Natural History University of Kansas 22:1-10. https://doi.org/10.5962/bhl.title.16290

Duellman W. E., L. Trueb \& E. Lehr. 2006. A new species of marsupial frog (Anura: Hylidae: Gastrotheca) from the Amazonian slopes of the Cordillera Oriental in Peru. Copeia 4: 595-603. https://doi.org/10.1643/00458511(2006)6[595:ANSOMF]2.0.CO;2

Duellman W. E., E. Lehr, D. Rodríguez, et al. 2004. Two new species of marsupial frogs (Anura: Hylidae: Gastrotheca) from the Cordillera Oriental in Central Peru. Occasional Papers of the Museum of Natural History University of Kansas 32: 1-10. https://doi.org/10.5962/bhl. part. 2471

Edgar R. C. 2004. MUSCLE: multiple sequence alignment with high accuracy and high throughput. Nucleic Acids Research 32 (5): 1792-97. https://doi.org/10.1093/ nar/gkh340

Fitzhugh K. 2005. The inferential basis of species hypotheses: the solution to defining the term "species". Marine Ecology, 26: 155-165. https//doi.org/10.1111/ j.1439-0485.2005.00058.x

Frost D. R. 2019. Amphibians Species of the World: an Online Reference. Versión 6.0 (acceso 24/09/2019). Museum of Natural History, New York, USA. http://research. amnh.org/herpetology/amphibia/index.html

Huang Y., J. Hu, B. Wang, et al. 2016. Integrative taxonomy helps to reveal the mask of the genus Gynandropaa (Amphibia: Anura: Dicroglossidae). Integrative Zoology 11: 134-150. https//doi.org/10.1111/1749-4877.12169. https://doi.org/10.1111/1749-4877.12169

Huelsenbeck J. P. \& F. Ronquist. 2001. MRBAYES: Bayesian inference of phylogeny. Bioinformatics 17:754-755. https://doi.org/10.1093/bioinformatics/17.8.754

IUCN SSC Amphibian Specialist Group 2018. Gastrotheca peruana. The IUCN Red List of Threatened Species 2018: (acceso 02/02/19). Disponible en línea:http:// dx.doi.org/10.2305/IUCN.UK.2018-1.RLTS. T55352A61397218.en.

Kumar S., k. Tamura \& M. Nei. 1994. MEGA: molecular evolutionary genetics analysis software for microcomputers. Computer Applications in the Biosciences 10 (2): 189-91. https://doi.org/10.1093/bioinformatics/10.2.189 
Lecocq T., N. J. Vereecken, D. Michez; et al. 2013. Patterns of genetic and reproductive traits differentiation in mainland vs. corsican populations of Bumblebees. PLoS ONE 8(6): e65642. https://doi.org/10.1371/journal. pone.0065642

Lehr E. \& A. Catenazzi. 2011. A new species of marsupial frog (Anura: Hemiphractidae: Gastrotheca) from the Río Abiseo National Park in Peru. Herpetologica 67 (4): 449-459. https://doi.org/10.1655/HERPETOLOGICA-D-11-00002.1

Luza A., G. L. Goncalves \& S. M. Hartz. 2015. Phylogenetic and morphological relationships between nonvolant small mammals reveal assembly at different spatial scales. Ecology and Evolution. 5 (4): 889-902 https//doi. org/10.1002/ece3.1407

McDiarmid R. W. 1994. Preparing amphibians as scientific specimens. Pp 289-296. In: Heyer W. E., M. A. Donnelly, R. W. McDiarmid, L. C. Hayek and M.S. Foster (eds.). Measuring and monitoring biological diversity. Standard methods for amphibians. Smithsonian Institution Press, Washington, 364 pp. +32 figures +28 tables

Martinez-Porchas M., E. Villalpando-Canchola, L. E. Ortíz-Suarez, et al. 2017. How conserved are the conserved 16S rRNA regions? PeerJ 5: 1-20 https//doi.org/10.7717/ peerj.3036

Miller M. A., W. Pfeiffer \& T. Schwartz. 2010. Creating the CIPRES Science Gateway for inference of large phylogenetic trees" in Proceeding of the Gateway Computing Environments Workshop (GCE), 14 Nov. 2010. New Orleans, LA: 1-8. https://doi.org/10.1109/ GCE.2010.5676129

Mitton J. B. 2001. Adaptation and natural selection. Overview. In eLS, (Ed). https://doi.org/10.1038/npg.els.0001706

Morales V. R., N. Carrillo \& H. Ortega. 1990. El material tipo de peces, anfibios y reptiles en el Museo de Historia Natural de la Universidad Nacional Mayor de San Marcos. Publicaciones del Museo de Historia Natural "Javier Prado", ser. A, Zoología 33:1-7.
Navas C. A., J. M. Carvajalino-Fernández, L. P. Saboyá-Acosta, et al. 2013. The body temperature of active amphibians along a tropical elevation gradient: patterns of mean and variance and inference from environmental data. Functional Ecology, 27: 1145-1154. https://doi. org/10.1111/1365-2435.12106

Song N., H. Li, F. Song, et al. 2016. Molecular phylogeny of Polyneoptera (Insecta) inferred from expanded mitogemic data. Scientific Reports 6:36175: 1-10. https://doi. org/10.1038/srep36175

Stamatakis A. 2014. RAxML, Version 8: A tool for Phylogenetic Analysis and Post-Analysis of Large Phylogenies. Bioinformatics, 2014:1-2. https://doi.org/10.1093/ bioinformatics/btu033

Trueb L. \& W. E. Duellman. 1978. An extraordinary new casqueheaded marsupial frog (Hylidae: Gastrotheca). Copeia 3: 498-503. https://doi.org/10.2307/1443617

Vellard J. 1957.Estudios sobre batracios andinos IV. El género Gastrotheca. Memorias del Museo de Historia Natural Javier Prado 5: 1-47.

Wang J., B. Lu, R. Zan, et al. 2016. Phylogenetic relationships of five Asian schilbid genera including Clupisoma (Siluriformes: Schilbeidae). PloS ONE 11(1): 1-16. https:// doi.org/10.1371/journal.pone.0145675

Wiens J. J., C. A. Kuczynski, W. E. Duellman, et al. 2007. Loss and revolution of complex life cycles in marsupial frogs: does ancestral trait reconstruction mislead? Evolution 61: 1886-1899. https://doi.org/10.1111/j.15585646.2007.00159.x

Yeates D. K., A. Seago, L. Nelson, et al. 2011. Integrative taxonomy, or iterative taxonomy? Systematic Entomology, 36: 209-217. https://doi.org/10.1111/j.13653113.2010.00558.x

\begin{abstract}
Agradecimientos:
Agradecemos a J. Córdova (MUSM), ex-Jefe del Departamento de Herpetología por el permiso para acceder y examinar los especímenes. Agradecemos a V. Correa, J. Sulca, J. Milla, D. Olivera, C. Ramírez, A. Ticona, J. Cusi, C. Torres, V. Herrera, L. Castillo, A. Mendoza y F. Huari por su asistencia en el trabajo de campo y colecta de nuevos especímenes. Un agradecimiento especial a M.Sc. Kenia Morales por su orientación en la obtención de las secuencias de ADN.
\end{abstract}

Conflicto de intereses:

Los autores no incurren en conflictos de intereses.

Rol de los autores:

DR concepción de la idea, trabajo de campo y procesamiento de especímenes, estandarización y ejecución de los protocolos, procesamiento de datos. GD estandarización y ejecución de los protocolos, procesamiento de datos. HO concepción de la idea. DR, GD, HO, CA revisaron y aprobaron el manuscrito.

Fuentes de financiamiento:

Financiado parcialmente con el proyecto FONDECYT 1180366 